\title{
Heterosis Studies for Seed Yield and its Component Traits in Indian Mustard [Brassica juncea (L.) Czern and Coss] Over Environments
}

\author{
Mahendar Singh Bhinda ${ }^{1 *}$, S. S. Shekhawat ${ }^{2}$, U. S. Shekhawat ${ }^{3}$ and A. K. Sharma ${ }^{2}$ \\ ${ }^{1}$ ICAR-Vivekananda Parvatiya Krishi Anusandhan Sansthan, Almora - 263601 \\ (Uttarakhand), India \\ ${ }^{2}$ Department of Genetics and Plant Breeding, S. K. Rajasthan Agricultural University, \\ Bikaner - 334 006, India \\ ${ }^{3}$ Agricultural Research Station (SKRAU), Sri Ganganagar - 335 001(Rajasthan), India \\ *Corresponding author
}

\section{A B S T R A C T}

\section{Keywords}

Indian mustard, Standard heterosis, Seed yield and Oil content

\section{Article Info}

Accepted:

28 July 2020

Available Online:

10 August 2020
Seventy five crosses of Indian mustard [Brassica juncea (L.) Czern \& Coss] generated by crossing of fifty lines with five testers in a line $\mathrm{x}$ tester mating design, which were used to estimate the standard heterosis potentiality for seed yield, its component traits and oil content. These parents, crosses and checks were sown in randomized complete block design under four environments each replicated thrice at two different locations. Observations were recorded on thirteen different characters. Standard heterosis was estimated on the basis of best check PUSA BOLD for these characters based on the pooled data over environments. The maximum values of standard heterosis recorded were $47.87 \%$ for seed yield per plant. The highest value of standard heterosis in case of yield components was $41.43 \%$ for harvest index; $34.01 \%$ for number of primary branches per plant; $31.59 \%$ for number of siliqua per plant; $27.03 \%$ for biological yield; $25.92 \%$ for 1000 -seed weight; $18.12 \%$ for number of seeds per siliqua; $17.21 \%$ for siliqua length; $13.79 \%$ for number of secondary branches per plant; $6.95 \%$ for plant height; $-17.00 \%$ for days to $50 \%$ flowering and $-8.85 \%$ for days to maturity. Standard heterosis results revealed that few hybrids viz., RH-30 x RGN-298, RL-1359 x RGN-298 and PBR-378 x Bio-902 were shown significant standard heterosis results for 10 or more characters towards desirable direction. The best three hybrids for seed yield per plant were Kranti x RGN-298 (47.87\%), RL-1359 x RGN-298 (47.53\%) and Kranti x RH-749 (43.98\%).

\section{Introduction}

Indian mustard [Brassica juncea (L.) Czern \& Coss] is an important Rabi season oilseed crop in India occupying a prestigious position among oilseed crops, which is popularly known as rai, raya or laha. It belongs to family (Brassicaceae) Crucifereae, the genus being Brassica. Cyto-genetically, Indian mustard is a natural amphidiploid $(2 n=36)$, derived from inter-specific hybridization between Brassica campestris $(2 \mathrm{n}=20)$ and Brassica nigra $(2 \mathrm{n}=16)$ followed by natural chromosome doubling of $\mathrm{F}_{1} \mathrm{~s}$. It is a naturally 
autogamous species in which out crossing varies from 5-30\% depending upon environmental conditions and frequency of pollinating insects (Shrimali et al., 2016). Brassica juncea is a crop of Asiatic origin with its major centre of diversity in China from where it was introduced in India (Vaughan, 1977). In India, it covers an area of 5.96 million hectares with 8.32 million tonnes production and $1397 \mathrm{~kg} \mathrm{ha}{ }^{-1}$ productivity and contributes nearly, 28.3 and 19.8 per cent as its share in acreage and production of rapeseed-mustard, respectively in the world (Anonymous, 2018).

The oil content in mustard seed ranges from 38-42 percent, which is yellow fragment and is considered to be the healthiest and nutritious cooking medium. Oil extracted from the seeds is used for cooking, frying, spice, for seasoning of the food articles, vegetables and industrial purposes.

Population of India is increasing rapidly and consequently edible oil demand is also going up day-by-day. Hence, it has become necessary to increase the production by developing superior varieties/hybrids. Heterosis breeding is an alternative tool which helps in sorting out probable gene combinations to overcome the existing yield barriers in the crop plants. Heterosis breeding in mustard has been recognized as a means of improving yield and other important traits. Therefore, knowledge regarding the magnitude and direction of heterosis is compelling need to exploit hybrid vigour commercially for increase and stabilize the production of Indian mustard. For acceptation of any hybrid for commercial cultivation, it must possess adequate superiority level over the standard/best check, which is referred as standard heterosis. In many Brassica spp. hybrid cultivars have been successful developed.
In the present study the intention of standard heterosis analysis was to recognize the best cross combinations which may provide high extent of economic heterosis for the concerned characters and depiction of their parents in order to utilization in future breeding programmes for hybrid development.

\section{Materials and Methods}

The material for present investigation was derived by crossing 15 varieties (lines) of Indian mustard with five testers viz., RGN236, RGN-298, RH-749, RLM-619 and Bio902 in a line $\mathrm{x}$ tester mating design. A set of seventy five crosses were evaluated along with twenty parents and 3 checks in randomized block design with three replications under two sets of environments $\mathrm{E}_{1}$ (normal) and $\mathrm{E}_{2}$ (moisture stress) at Instructional Research Farm of College of Agriculture, SKRAU, Bikaner and $\mathrm{E}_{3}$ (normal) and $\mathrm{E}_{4}$ (moisture stress) at Agricultural Research Station, Sri Ganganagar, separately during Rabi 2017-18.

Each genotype was sown as single row plot in $3 \mathrm{~m}$ length. Row to row and plant to plant spacing were kept at $45 \mathrm{~cm}$ and $15 \mathrm{~cm}$, respectively in each replication at both the locations. Observation were recorded for plant height $(\mathrm{cm})$, number of primary and secondary branches plant $^{-1}$, number of siliquae plant $^{-1}$, siliqua length $(\mathrm{cm})$, number of seeds per siliqua, 1000- seed weight $(\mathrm{g})$, biological yield plant ${ }^{-1}(\mathrm{~g})$, seed yield plant ${ }^{-1}$ $(\mathrm{g})$, harvest index $(\%)$ and oil content $(\%)$ on five randomly selected plant in each replication. The data on whole plot basis were recorded in case of days to $50 \%$ flowering and days to maturity.

To estimate the standard heterosis for all the characters including seed yield per plant PUSA BOLD was considered as the best 
standard check among the 3 checks taken for evaluation.

The experimental data recorded for various characters were analyzed as per the procedure of Panse and Sukhatme (1978) and standard heterosis was calculated following the method of Fonseca and Patterson (1968).

\section{Results and Discussion}

The analysis of variance for data pooled over environments revealed highly significant difference among genotypes, parents, crosses and between the environments for all the characters. This indicated the presence of adequate amount of genetic variability amongst the genotypes for all the characters which could be utilized for improvement, whereas environments selected for the study represented distinctly different climatic conditions.

Standard heterosis was computed for all the characters as per cent increase and decrease in mean performance of different crosses over the best check in the present experiment. The results of standard heterosis obtained over pooled data basis are presented in the Table 1 .

In the matters of superior performance for seed yield per plant along with component traits, the three best crosses were identified to be giving top performances on the basis of standard heterosis value, which are given in the Table 2.

\section{Days to $50 \%$ flowering}

In the experimental trial, fourteen crosses were found to be exhibiting significantly negative heterosis results suggesting towards their early flowering nature. The standard heterosis for days to $50 \%$ flowering ranged from -17.00 (Pusa Agrani x RGN-236) to 9.41 (MAYA $x$ RH-749). For days to $50 \%$ flowering, top three crosses, Pusa Agarni $\mathrm{x}$ RGN-236 (-17.00\%), Kranti x RGN-236 ($7.82 \%)$ and RGN-145 x Bio-902 (-7.36\%) have been identified as highly heterotic cross combinations with negatively significant standard heterosis values.

\section{Days to maturity}

Early maturity is useful in most of the plant species especially brassica where delayed maturity causes losses to yield and quality of oil due to rise in temperature; therefore, crosses exhibiting heterosis in negative direction are of immense value for earliness. The magnitude of standard heterosis results for days to maturity varied from -8.85 (Pusa Agrani x RGN-236) to 3.57 (RGN-303 x RGN-298).

The highest magnitude of standard heterosis was expressed by $-8.85 \%$ (Pusa Agrani $\mathrm{x}$ RGN-236) followed by $-7.29 \%$ (RN-393 x RLM-619) and $-7.11 \%$ (RGN-145 x RLM619).

Similar results were reported by Gupta and Narayan (2005), Monpara and Dobariya (2007), Vaghela et al., (2011), Patel et al., (2015) and Tomar et al., (2017) for days to $50 \%$ flowering and days to maturity from their studies on Indian mustard.

\section{Plant height}

In case of plant height, for which tallness has been reasoned as a requisite feature, the highest significant and positive standard heterosis results were reported by crosses viz., RH-30 x RGN-298 (6.95\%), RGN-145 x RGN-236 (5.76\%) and Kranti x RGN-298 $(5.39 \%)$. Variation of standard heterosis results for plant height falls between -20.01 (Pusa Agrani x RGN-236) to 6.95 (RH-30 x RGN-298). 
Table 1 Estimates of standard heterosis (SH) over best check (PUSA BOLD) on pooled data basis

\begin{tabular}{|c|c|c|c|c|c|c|c|c|c|c|c|c|c|c|}
\hline S.N. & Hybrids & $\begin{array}{c}\text { Days to } \\
50 \% \\
\text { flowering }\end{array}$ & $\begin{array}{l}\text { Days to } \\
\text { maturity }\end{array}$ & $\begin{array}{c}\text { Plant } \\
\text { height } \\
(\mathrm{cm})\end{array}$ & $\begin{array}{c}\text { No. of } \\
\text { primary } \\
\text { branches } \\
\text { per plant }\end{array}$ & $\begin{array}{c}\text { No. of } \\
\text { secondary } \\
\text { branches } \\
\text { per plant }\end{array}$ & $\begin{array}{l}\text { No. of } \\
\text { siliqua per } \\
\text { plant }\end{array}$ & $\begin{array}{l}\text { Siliqua } \\
\text { length } \\
(\mathrm{cm})\end{array}$ & $\begin{array}{c}\text { No. of } \\
\text { seeds per } \\
\text { siliqua }\end{array}$ & $\begin{array}{c}\text { 1000- } \\
\text { seed } \\
\text { weight } \\
(\mathrm{g})\end{array}$ & $\begin{array}{l}\text { Biological } \\
\text { yield per } \\
\text { plant } \\
\text { (g) }\end{array}$ & $\begin{array}{c}\text { Seed yield } \\
\text { per plant } \\
\text { (g) }\end{array}$ & $\begin{array}{c}\text { Harvest } \\
\text { index } \\
(\%)\end{array}$ & $\begin{array}{c}\text { Oil } \\
\text { content } \\
(\%)\end{array}$ \\
\hline 1 & Pusa Agarni x RGN-236 & $-17 * *$ & $-8.85 * *$ & $-20.01 * *$ & $-13.27 *$ & $-11.76^{*}$ & $-9.73 * *$ & $-29.01 * *$ & $-12.1 * *$ & $-23.98 * *$ & $-10.3^{*}$ & $-7.13^{*}$ & 3.25 & $-4.81 * *$ \\
\hline 2 & Pusa Agarni x RGN-298 & 3.55 & 0.16 & $-8.54 * *$ & 2.38 & -6.24 & $16.43^{* *}$ & $-13.35^{* *}$ & $-9.09 * *$ & $-10.64 *$ & -1.62 & $8.43^{* *}$ & $10.09 * *$ & $-3.82 * *$ \\
\hline 3 & Pusa Agarni x RH-749 & $-6.56^{*}$ & $-3.51^{*}$ & $-11.79 * *$ & $-14.63 * *$ & $-11.39 *$ & 7.01 & $-24.18 * *$ & $-26.29 * *$ & $-18.57 * *$ & $-9.37 *$ & 0 & $10.3 * *$ & $-8.01 * *$ \\
\hline 4 & Pusa Agarni x RLM-619 & -1.67 & -0.34 & $-9.73^{* *}$ & -3.23 & -10.23 & $-13.46^{* *}$ & $-26.69 * *$ & $-21.39 * *$ & $-18.18 * *$ & -5.41 & -1.05 & 5.13 & $-5.88 * *$ \\
\hline 5 & Pusa Agarni x Bio-902 & -3.87 & -1.09 & $-9.32 * *$ & 8.84 & 2.25 & $8.03^{*}$ & $-13.35 * *$ & $-7.06^{*}$ & -3.87 & 0.36 & $15.22 * *$ & $15.86^{* *}$ & $-7.56^{* *}$ \\
\hline 6 & Rohini x RGN-236 & -4.19 & $-3.38^{*}$ & -0.69 & 9.35 & -1.67 & $26.51^{* *}$ & -3.68 & 4.12 & 5.8 & -2.88 & $31.73^{* * *}$ & $35.87 * *$ & $-3.15^{* *}$ \\
\hline 7 & Rohini x RGN-298 & $-6.41 *$ & $-5.69 * *$ & -3.88 & 2.89 & -2.03 & $23.94 * *$ & $9.86^{* * *}$ & $12.88^{* *}$ & $10.64^{*}$ & 6.97 & $39.66^{* *}$ & $30.83 * *$ & $-6.42 * *$ \\
\hline 8 & Rohini x RH-749 & 4.67 & 2.76 & 0.82 & $10.71 *$ & -1.23 & -5.11 & $-17.79 * *$ & $-14.45^{* *}$ & -1.93 & $-9.91 *$ & 3.86 & $14.45^{* *}$ & $-7.19 * *$ \\
\hline 9 & Rohini x RLM-619 & 1.35 & -0.1 & $-10.51 * *$ & $11.22 *$ & -2.9 & $13.38 * *$ & $-29.01 * *$ & $-8.31 *$ & 2.32 & -1.08 & $9.97 * *$ & $10.73^{* *}$ & $-3.92 * *$ \\
\hline 10 & Rohini x Bio-902 & 3.72 & -2.21 & 0.41 & 4.42 & -0.87 & $10.88 * *$ & -3.29 & 2.03 & 6.77 & 4.68 & $21.67 * *$ & $16.42 * *$ & $-4.86^{* *}$ \\
\hline 11 & Kranti x RGN-236 & $-7.82 * *$ & $-3.88 *$ & 0.22 & -4.42 & -5.95 & $12.16^{* *}$ & $-8.51 * *$ & 2.09 & $11.22 *$ & 2.04 & $34.66^{* * *}$ & $31.47 * *$ & -1.09 \\
\hline 12 & Kranti x RGN-298 & -1.82 & -2.32 & 5.39 & $11.56^{*}$ & 0.15 & $31.59 * *$ & $17.21^{* *}$ & $17.85^{* *}$ & $25.92 * *$ & 4.62 & $47.87^{* *}$ & $41.26^{* *}$ & -0.84 \\
\hline 13 & Kranti x RH-749 & $-6.72 *$ & $-3.57 *$ & 0.09 & $10.71^{*}$ & -1.38 & $26.37 * *$ & $14.7 * *$ & $12.03 * *$ & $12.19 * *$ & 1.68 & $43.98 * *$ & $41.43 * *$ & $-1.96 * *$ \\
\hline 14 & Kranti x RLM-619 & 0.08 & -2.08 & $-8.91 * *$ & 0 & -2.9 & $14.75^{* * *}$ & $7.93 * *$ & $9.29 * *$ & $15.86^{* * *}$ & 1.26 & $32.84 * *$ & $30.74 * *$ & $-2.85^{* *}$ \\
\hline 15 & Kranti x Bio-902 & -3.87 & -2.39 & -0.14 & 3.23 & 2.32 & $19.64 * *$ & $11.8^{* *}$ & $10.46^{* *}$ & $16.83 * *$ & $8.29 *$ & $37.65^{* *}$ & $26.93 * *$ & $-5.7 * *$ \\
\hline 16 & RGN 303 X RGN-236 & 1.67 & 2.45 & 1.19 & 2.04 & -9.22 & $15.64 * *$ & $-7.74 * *$ & -3.07 & 6 & -7.21 & $15.9^{* * *}$ & $23.26^{* * *}$ & $-5.9 * *$ \\
\hline 17 & RGN 303 x RGN-298 & 5.14 & $3.57^{*}$ & $-6.72 *$ & -4.59 & -7.55 & 5.36 & $-11.03 * *$ & -5.04 & $17.99 * *$ & -2.16 & $9.63 * *$ & $11.12 * *$ & $-4.41 * *$ \\
\hline 18 & RGN $303 \times$ RH-749 & 3.4 & 1.83 & -3.15 & -6.63 & $-11.32 *$ & 7.12 & $-17.79 * *$ & $-10.07 * *$ & -4.84 & -3.78 & $7.38^{*}$ & $11.63 * *$ & $-8.26 * *$ \\
\hline 19 & RGN 303 x RLM-619 & 1.35 & 0.22 & 1.87 & -4.76 & -3.27 & 4.07 & $-13.93 * *$ & -2.88 & $9.09^{*}$ & 1.08 & $11.48^{* * *}$ & $9.66^{* *}$ & $-5.28 * *$ \\
\hline 20 & RGN 303 x Bio-902 & 1.67 & 0.77 & $-8.13^{* *}$ & 4.42 & -3.56 & $11.59 * *$ & $-12.57 * *$ & 6.34 & 2.71 & $12.07 * *$ & $13.86^{* * *}$ & 1.58 & -0.02 \\
\hline 21 & RN-393 x RGN-236 & $-6.18^{*}$ & $-4.94 * *$ & -5.34 & $12.41 *$ & 1.23 & $27.54 * *$ & $9.86^{* *}$ & $7.33^{*}$ & $24.18^{* *}$ & -0.72 & $39.07 * *$ & $39.89 * *$ & 0.1 \\
\hline 22 & RN-393 x RGN-298 & -0.74 & -2.51 & $-6.35^{*}$ & -5.61 & -5.95 & $18.47 * *$ & $9.28 * *$ & $10.99 * *$ & $10.64 *$ & 5.1 & $39.97 * *$ & $33.22 * *$ & $-6.57 * *$ \\
\hline 23 & RN-393 x RH-749 & -1.86 & -3.14 & -2.37 & $27.38^{* *}$ & 5.37 & $12.04 * *$ & $-8.12 * *$ & 0.65 & 8.32 & 1.01 & $35.4 * *$ & $34.42 * *$ & $-6.85^{* *}$ \\
\hline 24 & RN-393 x RLM-619 & -3.13 & $-7.29 * *$ & 1.14 & $12.07 *$ & 1.31 & $11.91^{* *}$ & $6.96^{*}$ & $7.65^{*}$ & $9.86^{*}$ & -1.84 & $31.67 * *$ & $34.54 * *$ & $-2.01 * *$ \\
\hline 25 & RN-393 x Bio-902 & -2.35 & $-4.94 * *$ & 3.52 & $18.2^{* *}$ & 6.75 & $9.15^{*}$ & $6.58 *$ & $6.87 *$ & 2.32 & -2.93 & $35 * *$ & $38.78^{* *}$ & $-1.51 * *$ \\
\hline 26 & PBR-357 x RGN-236 & 3.59 & $-3.26^{*}$ & $-10.8 * *$ & $-11.56^{*}$ & $-12.84 *$ & $-7.75^{*}$ & $-20.31 * *$ & $-6.87 *$ & $-13.93 * *$ & $-10.57 *$ & -5.28 & $5.81 *$ & $-5.23 * *$ \\
\hline 27 & PBR-357 x RGN-298 & 0.76 & $-4.87 * *$ & $-9.77 * *$ & 4.59 & -6.97 & $11.81^{* *}$ & $-8.7 * *$ & $6.47^{*}$ & $-11.22 *$ & 3.5 & $15.71 * *$ & $11.24 * *$ & $-4.91 * *$ \\
\hline 28 & PBR-357 x RH-749 & 4.55 & $-3.94 *$ & -4.37 & 4.93 & -7.76 & $19.48 * *$ & -1.35 & 1.57 & 0.97 & 5.87 & $10.56^{* * *}$ & 2.86 & $-7.34 * *$ \\
\hline 29 & PBR-357 x RLM-619 & 2.6 & -2.7 & $-9.64 * *$ & -1.36 & 1.31 & 6.57 & $-15.28 * *$ & $-10.2 * *$ & $-14.51 * *$ & $-9.97 *$ & 3.64 & $14.71 * *$ & $-3.92 * *$ \\
\hline 30 & PBR-357 x Bio-902 & $8.76^{* *}$ & 2.02 & -2.65 & $18.71 * *$ & 5.66 & 2.65 & $-7.35^{* *}$ & $9.55^{* *}$ & 1.93 & $10.8^{*}$ & $8.02 *$ & -1.28 & $-4.46^{* *}$ \\
\hline 31 & PBR-378 x RGN-236 & 2.37 & -0.53 & 0.87 & -0.85 & -6.39 & $20.47 * *$ & $-19.15^{* *}$ & $-7 *$ & $-11.99 * *$ & -3.67 & $9.69^{* *}$ & $13.38 * *$ & $-1.79 * *$ \\
\hline 32 & PBR-378 x RGN-298 & -3.4 & -3.14 & 4.87 & $26.53^{* *}$ & 4.28 & $31.31^{* *}$ & 4.84 & $7.33^{*}$ & $13.93^{* *}$ & 3.12 & $36.82 * *$ & $34.25^{* *}$ & $-1.29 *$ \\
\hline 33 & PBR-378 x RH-749 & -3.05 & -2.02 & -4.71 & $23.64 * *$ & 6.6 & $24.12 * *$ & 0.97 & 1.57 & -8.12 & -0.53 & $32.04 * *$ & $32.19 * *$ & $-2.6 * *$ \\
\hline 34 & PBR-378 x RLM-619 & $5.16^{*}$ & -0.16 & $-7.73^{* *}$ & $12.59^{*}$ & -5.66 & 4.09 & $-9.86^{* *}$ & -2.49 & 4.06 & -0.14 & $10.86^{* * *}$ & $9.66^{* * *}$ & $-4.19 * *$ \\
\hline 35 & PBR-378 x Bio-902 & -4.97 & $-6.55 * *$ & 5.21 & $34.01 * *$ & $13.79^{*}$ & $19.68 * *$ & $5.8^{*}$ & $12.43^{* *}$ & $18.57 * *$ & $16.47 * *$ & $37.07 * *$ & $18.55^{* *}$ & $-5.06^{* *}$ \\
\hline 36 & NPJ-112 x RGN-236 & 2.45 & 1.71 & $-7.1^{*}$ & 9.35 & -1.45 & $9.79^{* *}$ & -1.93 & 2.42 & 6.58 & $16.75^{* * *}$ & $15.99 * *$ & -1.2 & $-6.37 * *$ \\
\hline
\end{tabular}




\begin{tabular}{|c|c|c|c|c|c|c|c|c|c|c|c|c|c|c|}
\hline 37 & NPJ-112 x RGN-298 & $7.82 * *$ & -0.65 & -2.22 & 7.48 & -5.59 & 3.72 & -1.74 & $11.25^{* *}$ & $10.25^{*}$ & $15.38^{* * *}$ & 4.38 & $-9.41 * *$ & $-3.97 * *$ \\
\hline 38 & NPJ-112 x RH-749 & 4.97 & -2.27 & -4.85 & -4.08 & -5.81 & 6.42 & $-17.21 * *$ & $-7.52 *$ & -4.84 & 1.96 & 4.17 & 1.88 & $-3.87 * *$ \\
\hline 39 & NPJ-112 x RLM-619 & 2.69 & -2.08 & -4.43 & 5.61 & 3.34 & 7.13 & -5.03 & -0.98 & -1.55 & 2.64 & $8.77 * *$ & $6.63^{*}$ & $-1.41^{*}$ \\
\hline 40 & NPJ-112 x Bio-902 & -1.37 & $-3.57 *$ & $-12.96 * *$ & 0.17 & 0.36 & 3.21 & $-19.34 * *$ & -2.29 & -3.09 & -4.95 & $7.1 *$ & $14.24 * *$ & $-2.8 * *$ \\
\hline 41 & NPJ-113 x RGN-236 & 0.46 & -0.96 & $-17.18 * *$ & 0.85 & -5.59 & -0.6 & $-20.31 * *$ & -2.75 & 4.45 & 3.42 & 3.98 & 1.15 & $-3.2 * *$ \\
\hline 42 & NPJ-113 x RGN-298 & -2.41 & $-4.07 *$ & $-10.6^{* *}$ & -7.82 & -7.55 & 4.87 & $-29.98 * *$ & -4.84 & -4.84 & $-13.15^{* *}$ & $-8.15^{*}$ & 4.7 & $-1.66^{* *}$ \\
\hline 43 & NPJ-113 x RH-749 & 1.88 & -2.02 & $-5.58^{*}$ & 6.63 & 0.73 & 6.63 & $-13.93 * *$ & -1.18 & 8.32 & 3.42 & 5 & 0.86 & $-4.74 * *$ \\
\hline 44 & NPJ-113 x RLM-619 & $9.03 * *$ & 0.77 & $-5.81 *$ & -5.1 & $-13.57 *$ & 5.42 & $-9.28 * *$ & -2.09 & -0.39 & -2.16 & 2.16 & 3.33 & $-1.71 * *$ \\
\hline 45 & NPJ-113 x Bio-902 & 4.36 & -0.1 & -4.02 & $18.88^{* *}$ & 6.02 & $11.12^{* * *}$ & $-7.16^{* *}$ & 0.26 & $10.25^{*}$ & $20.18 * *$ & $16.11^{* *}$ & -2.61 & -0.64 \\
\hline 46 & MAYA x RGN-236 & $5.62 *$ & 1.33 & -3.79 & 0 & -7.55 & $9.11^{*}$ & $-20.5^{* *}$ & -1.18 & 0.97 & $10.27 *$ & $13.4^{* *}$ & 2.35 & $-3.7 * *$ \\
\hline 47 & MAYA x RGN-298 & 3.55 & -0.22 & -5.07 & 1.87 & -4.21 & -3.65 & $-28.43 * *$ & $-7.26^{*}$ & $-13.35 * *$ & $-9.73 *$ & -5.09 & 4.66 & $-3.4 * *$ \\
\hline 48 & MAYA x RH-749 & $9.41^{* *}$ & -2.76 & 2.33 & -2.21 & -3.63 & $10.59^{* *}$ & $-13.54 * *$ & -2.29 & 0.39 & $22.4 * *$ & $8.21^{*}$ & $-11.2 * *$ & $-5.16^{* *}$ \\
\hline 49 & MAYA x RLM-619 & $6.56^{*}$ & -0.47 & 1.1 & $15.82^{* *}$ & 5.44 & 1.84 & $-20.5^{* *}$ & -3.73 & -1.55 & -6.67 & -2.25 & 5.26 & $-6.3 * *$ \\
\hline 50 & MAYA x Bio-902 & 2.92 & 2.21 & -0.69 & 9.18 & -1.67 & 4.1 & $-15.86^{* *}$ & 1.18 & $12.77 * *$ & $27.03^{* * *}$ & $9.85^{* *}$ & $-12.95 * *$ & $-3.55^{* *}$ \\
\hline 51 & RGN-145 x RGN-236 & -2.6 & $-4.75^{* * *}$ & $5.76^{*}$ & $22.28 * *$ & 3.05 & $19.63^{* *}$ & 5.22 & $18.12 * *$ & 2.51 & $22.16^{* * *}$ & $36.45^{* *}$ & $12.44 * *$ & $-2.43 * *$ \\
\hline 52 & RGN-145 x RGN-298 & -4.02 & $-4.01 *$ & -3.34 & $17.18^{* *}$ & 5.66 & $28.12^{* *}$ & $-7.16^{* *}$ & $6.87^{*}$ & 2.9 & $10.15^{*}$ & $37.47 * *$ & $26.08^{* *}$ & $-2.16^{* *}$ \\
\hline 53 & RGN-145 x RH-749 & -4.52 & $-5.56^{* * *}$ & $-6.4 *$ & $17.18^{* *}$ & 2.9 & $25.08^{* *}$ & $5.8^{*}$ & $7.85^{*}$ & 0.39 & $8.65^{*}$ & $35.46^{* *}$ & $29.12 * *$ & $-3.62 * *$ \\
\hline 54 & RGN-145 x RLM-619 & $-5.29 *$ & $-7.11^{* * *}$ & 0.36 & $14.46^{* *}$ & 6.97 & $12.72 * *$ & 3.29 & $14.85^{* *}$ & $12.19^{* *}$ & $16.58^{* * *}$ & $37.07 * *$ & $19.75^{* * *}$ & $-4.59 * *$ \\
\hline 55 & RGN-145 x Bio-902 & $-7.36^{* *}$ & -3.08 & $-6.12^{*}$ & $10.71^{*}$ & 4.35 & $15.57^{* *}$ & $6.38^{*}$ & $8.7 * *$ & 8.32 & 6.49 & $37.19^{* *}$ & $28.73^{* *}$ & -0.45 \\
\hline 56 & RL-1359 x RGN-236 & $-5.46^{*}$ & $-5.93 * *$ & $-10.37 * *$ & $-11.39 *$ & -6.97 & 4.58 & $-7.16^{* * *}$ & -5.89 & -6.58 & 7.93 & 0.86 & $-5.94 *$ & $-4.49 * *$ \\
\hline 57 & RL-1359 x RGN-298 & $-5.71 *$ & $-5.12 * *$ & 4.29 & $17.86^{* *}$ & 4.28 & $25.27 * *$ & $11.41 * *$ & $9.74 * *$ & $10.25^{*}$ & $12.43 * *$ & $47.53 * *$ & $32.28 * *$ & $-3.1 * *$ \\
\hline 58 & RL-1359 x RH-749 & -3.51 & $-4.13 *$ & $-14.3^{* * *}$ & 2.55 & -7.84 & 5.22 & $-11.61 * *$ & $-23.41 * *$ & -4.84 & 2.52 & 1.79 & -1.03 & $-5.43 * *$ \\
\hline 59 & RL-1359 x RLM-619 & $-5.62 *$ & $-5.8 * *$ & -3.33 & -3.4 & 2.69 & $15.77 * *$ & 1.16 & $9.68 * *$ & 0 & $16.76^{* * *}$ & $36.82 * *$ & $18.17 * *$ & $-3.15^{* *}$ \\
\hline 60 & RL-1359 x Bio-902 & $6.89 * *$ & 1.83 & 0.32 & 0.51 & -5.37 & 2.73 & -2.13 & 6.41 & $-16.05 * *$ & -2.04 & $8.92 * *$ & $12.95^{* * *}$ & $-6.4 * *$ \\
\hline 61 & RGN-73 x RGN-236 & 1.56 & -2.7 & $-13.84 * *$ & -0.17 & -2.54 & 4.79 & $-23.4 * *$ & $-8.11 *$ & $-19.34 * *$ & $-9.37 *$ & 4.69 & $12.91 * *$ & -0.62 \\
\hline 62 & RGN-73 x RGN-298 & -4.97 & $-4.5^{* *}$ & $-10.05 * *$ & 1.7 & 5.01 & $17.84^{* * *}$ & $-10.64 * *$ & $9.74 * *$ & -4.84 & -4.02 & $33.7 * *$ & $38.82 * *$ & $-4.64 * *$ \\
\hline 63 & RGN-73 x RH-749 & $5.44 *$ & -2.33 & $-15.39 * *$ & -5.44 & -2.25 & 6.82 & $-22.05 * *$ & -5.04 & $-17.41 * *$ & -1.8 & -1.3 & -0.43 & $-6.6^{* *}$ \\
\hline 64 & RGN-73 x RLM-619 & -4.34 & $-3.45^{*}$ & $-10.69 * *$ & $14.63^{* *}$ & -4.93 & $11.12^{* *}$ & $-13.15 * *$ & 1.31 & $-8.9^{*}$ & 5.95 & $15.86^{* *}$ & $8.55^{* *}$ & $-4.86 * *$ \\
\hline 65 & RGN-73 x Bio-902 & -1.97 & $-3.26^{*}$ & -1.33 & $15.14 * *$ & 4.06 & $27.97 * *$ & $6.38 *$ & $10.73 * *$ & $10.25 *$ & $12.97 * *$ & $36.45^{* *}$ & $20.31 * *$ & $-3.67 * *$ \\
\hline 66 & Varuna x RGN-236 & -0.08 & -1.96 & $-6.21 *$ & 1.19 & -0.73 & -2.82 & $-11.99 * *$ & -3.07 & $-11.61 * *$ & -5.05 & 4.32 & $8.51^{* *}$ & 0.77 \\
\hline 67 & Varuna x RGN-298 & -1.67 & -1.77 & -5.21 & 3.74 & -7.55 & $11.31 * *$ & $-6.19^{*}$ & -5.43 & -7.35 & 1.98 & $7.13^{*}$ & 4.4 & $-6.6 * *$ \\
\hline 68 & Varuna x RH-749 & 2.45 & -0.1 & $-8.36^{* *}$ & $10.2 *$ & 2.32 & $7.65^{*}$ & 0.39 & 2.16 & -1.93 & 7.39 & $12.04 * *$ & 3.76 & $-3.42 * *$ \\
\hline 69 & Varuna x RLM-619 & -0.72 & -3.14 & $-7.59 * *$ & $10.71 *$ & -2.39 & 5.19 & $-5.8 *$ & $6.87^{*}$ & -2.9 & 2.52 & $9.32^{* *}$ & $6.03^{*}$ & $-1.84 * *$ \\
\hline 70 & Varuna x Bio-902 & $9.39 * *$ & 1.89 & 0.14 & $15.65^{* *}$ & 7.4 & $21.81^{* *}$ & 5.22 & $12.95^{* *}$ & 0.39 & $16.22 * *$ & $20.12^{* *}$ & 2.22 & $-3.32 * *$ \\
\hline 71 & RH-30 x RGN-236 & $-5.29 *$ & $-4.75^{* * *}$ & 1.51 & 6.8 & -1.89 & $24.58^{* *}$ & -1.55 & 5.3 & 3.48 & -2.7 & $31.42^{* *}$ & $35.66^{* *}$ & -0.72 \\
\hline 72 & RH-30 x RGN-298 & $-6.09 *$ & $-5.31 * *$ & $6.95^{*}$ & $16.84 * *$ & 6.6 & $20.02 * *$ & $10.83^{* * *}$ & $15.04 * *$ & $11.61 * *$ & $10.45^{*}$ & $37.1 * *$ & $24.45^{* * *}$ & $-1.74 * *$ \\
\hline 73 & RH-30 x RH-749 & 1.29 & -2.08 & -2.97 & 0.51 & -2.18 & -4.61 & $-20.7 * *$ & $-8.18^{*}$ & $-14.51 * *$ & -0.54 & 6.23 & $7.48^{* *}$ & $-5.63 * *$ \\
\hline 74 & RH-30 x RLM-619 & 0.72 & -1.27 & $-5.58 *$ & $12.59^{*}$ & 2.1 & $10.19^{* *}$ & -0.97 & $7.33^{*}$ & 2.51 & 5.59 & $15.46^{* *}$ & $8.42 * *$ & $-3.89 * *$ \\
\hline 75 & RH-30 x Bio-902 & $-5.24 *$ & -2.45 & 2.15 & $19.73^{* *}$ & 6.53 & $23.8^{* *}$ & $15.09 * *$ & $16.74 * *$ & $12.38^{* * *}$ & $14.41 * *$ & $42.87^{* *}$ & $26.38^{* *}$ & 0.77 \\
\hline \multicolumn{15}{|c|}{$*, * *$ Significant at $5 \%$ and $1 \%$ level of significance, respectively } \\
\hline
\end{tabular}


Table.2 Top three performing crosses on the basis of standard heterosis values for seed yield and component traits

\begin{tabular}{|c|c|c|c|}
\hline Characters & \multicolumn{3}{|c|}{ Crosses } \\
\hline Days to $50 \%$ flowering & $\begin{array}{l}\text { Pusa Agrani x RGN- } \\
236(-17.00 \%)\end{array}$ & $\begin{array}{c}\text { Kranti x RGN-236 } \\
(-7.82 \%)\end{array}$ & $\begin{array}{l}\text { RGN-145 x Bio-902 } \\
(-7.36 \%)\end{array}$ \\
\hline Days to maturity & $\begin{array}{l}\text { Pusa Agrani x RGN- } \\
236(-8.85 \%)\end{array}$ & $\begin{array}{c}\text { RN-393 x RLM-619 } \\
(-7.29 \%)\end{array}$ & $\begin{array}{c}\text { RGN-145 x RLM-619 } \\
(-7.11 \%)\end{array}$ \\
\hline Plant height (cm) & $\begin{array}{l}\text { RH-30 x RGN-298 } \\
(6.95 \%)\end{array}$ & $\begin{array}{c}\text { RGN-145 x RGN-236 } \\
(5.76 \%)\end{array}$ & $\begin{array}{c}\text { Kranti x RGN-298 } \\
(5.39 \%)\end{array}$ \\
\hline $\begin{array}{c}\text { Number of primary branches per } \\
\text { plant }\end{array}$ & $\begin{array}{l}\text { PBR-378 x Bio-902 } \\
\quad(34.01 \%)\end{array}$ & $\begin{array}{c}\text { RN-393 x RH-749 } \\
(27.38 \%)\end{array}$ & $\begin{array}{c}\text { PBR-378 x RGN-298 } \\
(26.53 \%)\end{array}$ \\
\hline $\begin{array}{c}\text { Number of secondary branches per } \\
\text { plant }\end{array}$ & $\begin{array}{l}\text { PBR-378 x Bio-902 } \\
\quad(13.79 \%)\end{array}$ & $\begin{array}{c}\text { Varuna } x \text { Bio-902 } \\
(7.40 \%)\end{array}$ & $\begin{array}{c}\text { RGN-145 x RLM-619 } \\
(6.97 \%)\end{array}$ \\
\hline Number of siliqua per plant & $\begin{array}{c}\text { Kranti x RGN-298 } \\
\text { (31.59\%) }\end{array}$ & $\begin{array}{c}\text { PBR-378 x RGN-298 } \\
(31.31 \%)\end{array}$ & $\begin{array}{c}\text { RGN }-145 \times \text { RGN-298 } \\
(28.12 \%)\end{array}$ \\
\hline Siliqua length $(\mathrm{cm})$ & $\begin{array}{c}\text { Kranti x RGN-298 } \\
(17.21 \%)\end{array}$ & $\begin{array}{c}\text { RH-30 x Bio-902 } \\
(15.09 \%)\end{array}$ & $\begin{array}{c}\text { Kranti x RH-749 } \\
(14.70 \%)\end{array}$ \\
\hline Number of seeds per siliqua & $\begin{array}{c}\text { RGN-145 x RGN-236 } \\
(18.12 \%)\end{array}$ & $\begin{array}{l}\text { Kranti x RGN-298 } \\
\quad(17.85 \%)\end{array}$ & $\begin{array}{l}\text { RH-30 x Bio-902 } \\
\quad(16.74 \%)\end{array}$ \\
\hline 1000- seed weight (g) & $\begin{array}{c}\text { Kranti x RGN-298 } \\
(25.92 \%)\end{array}$ & $\begin{array}{l}\text { RN-393 x RGN-236 } \\
(24.18 \%)\end{array}$ & $\begin{array}{c}\text { PBR-378 x Bio-902 } \\
(18.57 \%)\end{array}$ \\
\hline Biological yield per plant (g) & $\begin{array}{l}\text { MAYA x Bio-902 } \\
(27.03 \%)\end{array}$ & $\begin{array}{l}\text { MAYA x RH-749 } \\
(22.40 \%)\end{array}$ & $\begin{array}{c}\text { RGN-145 x RGN-236 } \\
(22.16 \%)\end{array}$ \\
\hline Seed yield per plant (g) & $\begin{array}{c}\text { Kranti x RGN-298 } \\
(47.87 \%)\end{array}$ & $\begin{array}{c}\text { RL-1359 x RGN-298 } \\
(47.53 \%)\end{array}$ & $\begin{array}{c}\text { Kranti x RH-749 } \\
(43.98 \%)\end{array}$ \\
\hline Harvest index (\%) & $\begin{array}{c}\text { Kranti x RH-749 } \\
(41.43 \%)\end{array}$ & $\begin{array}{c}\text { Kranti x RGN-298 } \\
(41.26 \%)\end{array}$ & $\begin{array}{c}\text { RN-393 x RGN-236 } \\
(39.89 \%)\end{array}$ \\
\hline Oil content $(\%)$ & $\begin{array}{c}\text { RH-30 x Bio-902 } \\
(0.77 \%)\end{array}$ & $\begin{array}{c}\text { Varuna x RGN-236 } \\
(0.77 \%)\end{array}$ & $\begin{array}{c}\text { RN-393 x RGN-236 } \\
(0.1 \%)\end{array}$ \\
\hline
\end{tabular}

Number of primary and secondary branches per plant

The standard heterosis results for number of primary branches per plant varied from -14.63 (Pusa Agrani x RH-749) to 34.01 (PBR-378 x Bio-902); whereas for number of secondary branches per plant ranged between -13.57 (NPJ113 x RLM-619) to 13.79 (PBR-378 x Bio902). For number of primary branches per plant and number of secondary branches per plant, PBR-378 x Bio-902 was reported the best standard heterotic cross with values of $34.01 \%$ and $13.79 \%$ respectively, followed by RN-393 x RH-749 (27.38\%), PBR-378 x RGN-298 (26.53\%) for number of primary branches per plant and Varuna x Bio-902 (7.40\%), RGN-145 x RLM-619 (6.97\%) for number of secondary branches per plant. Findings of similar nature were reported by Monpara and Dobariya (2007), Kumar et al., (2013) and Tomar et al., (2017).

\section{Number of siliqua per plant}

Standard heterosis results for number of siliqua per plant ranged from -13.46 (Pusa Agrani x RLM-619) to 31.59 (Kranti x RGN-298). For number of siliqua per plant the highest percentage of improvement in performance over the best check variety was reported by Kranti $\mathrm{x}$ RGN-298 (31.59\%) followed by PBR-378 x 
RGN-298 (31.31\%) and RGN -145 x RGN-298 (28.12\%) depicting the superiority of these crosses for this character. These findings are similar to the one reported by Aher et al., (2009), Patel et al., (2015) and Shrimali et al., (2018).

\section{Siliqua length}

Estimates of standard heterosis for siliqua length ranged from -29.98 (NPJ-113 x RGN298) to 17.21 (Kranti x RGN-298). Kranti $x$ RGN-298 reported maximum standard heterosis results for siliqua length with value of 17.21 showing a significant increase over the best check followed by RH-30 x Bio-902 (15.09\%) and Kranti x RH-749 (14.70\%). Similar findings were reported by Teklewood and Becker (2005), Monpara and Dobariya (2007), Adhikari et al., (2017) and Kumar et al., (2018) for siliqua length.

\section{Number of seeds per siliqua}

The magnitudes of standard heterosis results for number of seeds per siliqua varied from -23.41 (RL-1359 x RH-749) to 18.12 (RGN-145 x RGN-236). The highest standard heterosis results for number of seeds per siliqua have been reported by crosses viz., RGN-145 x RGN-236 (18.12\%) followed by Kranti x RGN$298(17.85 \%)$ and RH-30 x Bio-902 (16.74\%). These findings have also been substantiated by the findings of Prajapati et al., (2007), Kumar et al., (2013), and Kumar et al., (2018) as they also found moderate to low level of positive heterosis for number of seeds per siliqua which directly adds to improve seed yield per plant.

\section{0- Seed weight}

The results for estimates of standard heterosis ranged between -23.98 (Pusa Agarni x RGN236) to 25.92 (Kranti x RGN-298). Kranti $x$ RGN-298 reported maximum standard heterosis results for 1000-seed weight with value of $17.21 \%$ followed by RN-393 x RGN-236 (24.18\%), PBR-378 x Bio-902 (18.57\%). The low to moderate level of heterosis for 1000 -seed weight was also observed by Monpara and Dobariya (2007), Prajapati et al., (2007) and Patel et al., (2015).

\section{Biological yield per plant}

Estimates of standard heterosis for biological yield per plant ranged from -13.15 (NPJ-113 x RGN-298) to 27.03 (MAYA x Bio-902). For biological yield per plant, the highest standard heterosis results was reported for MAYA x Bio$902(27.03 \%)$ followed by MAYA x RH-749 $(22.40 \%)$ and RGN-145 x RGN-236 (22.16\%) indicating towards their superiority over various other crosses in the matter of biological yield per plant. These findings are similar to the one reported by Kumar et al., (2014) and Kumar et al., (2018).

\section{Seed yield per plant}

The magnitudes of standard heterosis results for seed yield per plant ranged from -7.13 (Pusa Agrani x RGN-236) to 47.87 (Kranti x RGN298). In case of seed yield per plant, the highest standard heterosis effects was reported for Kranti x RGN-298 with the magnitude of $47.87 \%$ followed by RL-1359 x RGN-298 (47.53\%) and Kranti x RH-749 (43.98\%).

Similar to this finding, moderate to high heterosis and significantly positive results for seed yield per plant have also been reported by Prajapati et al., (2007), Aher et al., (2009), Vaghela et al., (2011), Kumar et al., (2013), Tomar et al., (2014), Patel et al., (2015), Meena et al., (2017) and Shrimali et al., (2018).

\section{Harvest index}

Estimates of heterosis for harvest index varied between -12.95 (MAYA x Bio-902) to 41.43 (Kranti x RH-749) over the best check. For the harvest index, crosses, namely, Kranti x RH749 (41.43\%), Kranti x RGN-298 (41.26\%) and RN-393 x RGN-236 (39.89\%) were reported as the best performing crosses on the basis of standard heterosis results. These findings have been substantiated by the findings of Prajapati 
et al., (2007) and Shrimali et al., (2018) since, they also reported moderate amount of positive hetrosis for harvest index.

\section{Oil content}

Standard heterosis results for oil content were found to be significantly negative for many hybrids, where positively significant heterosis for oil content would have been a desirable feature. Nonetheless, a small number of crosses have reported non significant but positive standard heterosis for this trait viz., RH-30 x Bio-902 (0.77\%), Varuna x RGN-236 $(0.77 \%)$ and RN-393 x RGN-236 (0.1\%). Results of this nature for oil content have also been reported by Prajapati et al., (2007), Vaghela et al., (2011) and Kumar et al., (2014) as low levels of non significant heterosis for oil content were registered in many crosses by them.

In conclusion the significant heterosis for seed yield was the result of combined effect of other contributing traits therefore; the selection of high yielding genotypes should be based on multiple characters rather than a single character. Estimates of heterotic responses further showed the perceptible advantage of heterozygosity in improving the seed yield. This phenomenon led to identify heterosis breeding as the key methodology for improving genetic yield ceiling in Indian mustard.

In almost all the characters, variable number of crosses depicted standard heterosis in both positive and negative direction, indicating that genes with negative as well as positive effects were dominant in the experimental material under study. Similar finding for various characters in Indian mustard were also earlier reported by Gami and Chauhan (2013), Meena et al., (2014) and Patel et al., (2015). This show of unpredictability highlights the role of non additive gene actions, which in turn may be due to dominance or/and epistasis effects. Therefore, these genotypes may be used in the future breeding programme for development of mustard hybrids suitable for varying environments in order to maximize mustard production.

\section{References}

Adhikari, S., Pathak, S., Joshi, D., Pant, U., Singh, A.K. and Bhajan, R. (2017). Heterosis analysis for seed yield and other component traits in Indian mustard [Brassica juncea (L.) Czern and Coss]. Int. J. Curr. Microbiol. App. Sci., 6(10): 1157-1162.

Aher, C.D., Shelke, L.T., Chinchane, V.N., Borgaonkar, S.B., and Gaikwad, A.R. (2009). Heterosis for yield and yield components in Indian mustard [Brassica juncea (L.) Czern \& Coss]. International Journal of Plant Sciences, 4(1): 30-32.

Anonymous (2018). Agricultural Statistics at A Glance, Directorate of Economics and Statistics, Ministry of Agriculture \& Farmers Welfare, Govt. of India.

Fonseca, S. and Patterson. F.L. (1968). Hybrid vigour in a seven parent diallel cross in common winter wheat (Triticum aestivum L.). Crop Sci., 8: 85-88.

Gami, R.A. and Chauhan, R.M. (2013). Heterosis and combining ability analysis for seed yield and its attributes in Indian mustard [Brassica juncea (L.) Czern and Coss]. Internat. J. Agric. Res., 47(6): 535-539.

Gupta, P.C. and Narayan, S. (2005). Potential crosses for development of hybrid varieties in Indian mustard (B. juncea). $J$ Oilseeds Res., 22: 164-167.

Kumar, B., Pandey, A. and Kumari, A. (2018). Heterosis and combining ability of $\mathrm{F}_{1}$ and $\mathrm{F}_{2}$ generations of Indian mustard for seed yield and its attributes. Journal of Oilseed Brassica, 9(1): 33-39.

Kumar, B., Pandey, A. and Singh, S.K. (2014). Combining ability and economic heterosis for yield and quality traits in Indian mustard [Brassica juncea (L.) Czern \& Coss]. Electronic Journal of Plant Breeding, 5(2): 203-207.

Kumar, P., Lamba, A., Yadav, R.K., Singh, L. and Singh, M. (2013). Analysis of yield and its components based on heterosis and combining ability in Indian mustard [Brassica juncea (L.) Czern \& Coss]. The 
Bioscan, 8(4): 1497-1502.

Meena, H.S., Kumar, A., Kulshrestha, S., Meena, P.D., Ram, B., Sharma, A., Singh, V.V. and Singh, S. (2017). Line x tester analysis for combining ability and heterosis in Indian mustard (Brassica juncea). Journal of Oilseed Brassica, 8(1): 18-26.

Meena, H.S., Ram, B., Kumar, A., Singh, B.K., Meena, P.D., Singh, V.V. and Singh, D. (2014). Heterobeltiosis and standard heterosis for seed yield and important traits in Brassica juncea. Journal of Oilseed Brassica, 5(2): 134-140.

Monpara, B. A. and Dobariya, K. L. (2007). Heterosis and combining ability in Indian mustard. J. Oilseeds Res., 24(2): 306-308.

Panse, V.G. and Sukhatme, P.V. (1985). Statistical Methods for Agricultural Workers. I.C.A.R., New Delhi.

Patel, R., Solanki, S.D., Gami, R.A., Prajapati, K.P., Patel, P.T. and Bhadauria, H.S. (2015). Genetic study for seed yield and seed quality traits in Indian mustard. Electronic Journal of Plant Breeding , 6(3): 672-679.

Prajapati, C.H., Patel, K.M., Patel, M.P. and Pathak, H.C. (2007). Heterosis for seed yield and its components in Indian rapeseed, (Brassica campestris ssp. yellow sarson). Journal of Oilseeds Research, 24: 309-310.

Shrimali, T.M., Chauhan, R.M., Gami, R.A. and
Patel, P.T. (2016). Diallel analysis in Indian mustard (Brassica juncea L. Czern \& Coss.). Electronic Journal of Plant Breeding, 7(4): 919-924.

Shrimali, T.M., Chauhan, R.M., Prajapati, K.P., Desai, S.A., Patel, J.R., Patel, P.T., Patel, P.J. and Chaudhary, B.K. (2018). Analysis of yield and its components based on heterosis and combining ability in Indian mustard (Brassica juncea L. Czern \& Coss.). Int. J. Pure App. Biosci., 6(1): 219-224.

Teklewold, A. and Becker, H.C. (2005). Heterosis and combining ability in a diallel cross of Ethiopian mustard inbred lines. Crop Science, 45: 2629-2635.

Tomar, A., Singh, M. and Singh, S.K. (2017). Combining ability (SCA \& GCA) and heterotic response analysis in Indian mustard (Brassica juncea L. Czern \& Coss). Journal of Pharmacognosy and Phytochemistry, 6(4): 1999-2004.

Vaghela, P.O., Thakkar, D.A., Bhadauria, H.S., Sutariya, D.A., Parmar, S.K. and Prajapati, D.V. (2011). Heterosis and combining ability for yield and its component traits in Indian mustard (Brassica juncea L.). Journal of Oilseed Brassica, 2(1): 39-43.

Vaughan, J.G. (1977). Multidisciplinary study of the taxonomy and origin of Brassica crops. Bio-Science, 27(1): 35-40.

\section{How to cite this article:}

Mahendar Singh Bhinda, S. S. Shekhawat, U. S. Shekhawat and Sharma, A. K. 2020. Heterosis Studies for Seed Yield and its Component Traits in Indian Mustard [Brassica juncea (L.) Czern and Coss] Over Environments. Int.J.Curr.Microbiol.App.Sci. 9(08): 3818-3826. doi: https://doi.org/10.20546/ijcmas.2020.908.440 